\title{
Room-Temperature Self-Assembly of Equilateral Triangular Clusters via Friedel Oscillations
}

\author{
G. Manai, ${ }^{1, *}$ K. Radican, ${ }^{1}$ F. Delogu, ${ }^{2}$ and I. V. Shvets ${ }^{1}$ \\ ${ }^{1}$ School of Physics, CRANN, Trinity College, Dublin 2, Ireland \\ ${ }^{2}$ Dipartimento di Ingegneria Chimica e Materiali, Università degli Studi di Cagliari, Piazza d'Armi, I-09123 Cagliari, Italy
}

(Received 30 May 2008; revised manuscript received 24 July 2008; published 14 October 2008)

\begin{abstract}
We report on the formation of equilateral triangular clusters hollow inside with 5-6 atoms per side, selfassembled on $\mathrm{Ni}$ adislands grown on $\mathrm{Rh}(111)$. The observation of standing wave patterns on the $\mathrm{Ni}$ adislands and the $\mathrm{Rh}(111)$ indicates that the self-assembly is mediated by Friedel oscillations. In this context, we propose a model based on the energy of interaction between adsorbates, which explains the formation of the clusters as a result of the assembly of rows of 5-6 adatoms.
\end{abstract}

DOI: 10.1103/PhysRevLett.101.165701

Much of the current scientific research is dedicated to the fabrication of well-defined structures in the atomic range. Gaining control at the nanoscale of the geometric, electronic and magnetic properties of existing and new materials is one of the most important goals of nanoscience and nanotechnology. Atom manipulation [1], self-assembly [2] and self-organization [3] are the main available tools for building nanostructures.

Several studies of self-assembly on the (111) surface of noble metals, have shown promising results towards the formation of long-range superstructures, although they are generally only stable at a very low temperature (LT) [4-7]. The necessary ingredient for this type of self-organization is the presence of a Shockley surface state $[8,9]$. In these conditions, scattering of the electrons by a charge defect gives rise to surface Friedel oscillations [10]. At the Fermi energy $\left(E_{F}\right)$, due to the discontinuity in the electrons' momentum, interference of Friedel oscillations can generate standing waves (SWs) of wavelength $\lambda_{F} / 2=\pi / k_{F}$ [11]. The SW modifies the local density of states (LDOS) at the surface, and can therefore be imaged directly by scanning tunneling microscopy and spectroscopy (STM-STS) $[10,12,13]$. Moreover, the change in the LDOS affects the adsorption energy of surface adsorbates, that tend to occupy regions of high LDOS.

In this Letter we describe the room-temperature (RT) self-assembly of hollow equilateral triangular nanoclusters with a narrow size distribution centered around clusters of either 5 or 6 atoms per side. The clusters form on top of submonolayer-thick Ni films supported on a Rh(111) substrate. We speculate that the reason for the stability of the clusters at RT is the enhancement of the interference between Friedel oscillations confined to the Ni adislands.

Epitaxial Ni ultrathin films of thickness below 1 monolayer (ML), where $1 \mathrm{ML}$ is defined as a layer of atoms in a hexagonal lattice with unit $a_{\mathrm{Rh}}=2.69 \AA$, were grown on a $\mathrm{Rh}(111)$ substrate. For each coverage, namely, 0.1, 0.25, $0.5,0.8$, and 1.0 MLs, an experiment was performed. Then, the Ni film was removed by prolonged $\mathrm{Ar}^{+}$sputtering and annealing at $1075 \mathrm{~K}$. The cleanliness of the surface was
PACS numbers: 64.75.Yz, 61.46.Bc, 68.37.Ef, 73.20.Hb

checked with Auger electron spectroscopy (AES) and showed no elements on the surface other than Rh. At all coverages, LEED data exhibited a sharp hexagonal pattern indicating a $1 \times 1$ registry between the film and the substrate, in agreement with previous studies [14]. The lattice parameter for the relaxed $\mathrm{Ni}(111)$ is $a_{\mathrm{Ni}}=2.49 \AA$, giving a mismatch strain of $7.4 \%$.

STM images show that Ni decorates the steps of the Rh (111) substrate and also forms adislands of triangular shape, coherently oriented with sides parallel to the [011], [110] and [101] directions, see Fig. 1(a). The formation of oriented triangular islands, and not hexagonal ones, is most likely due to the presence of a strain field induced by the step edges, which modifies diffusion along preferential directions [15], although the possibility that the growth is affected by $\mathrm{CO}$ contamination cannot be fully

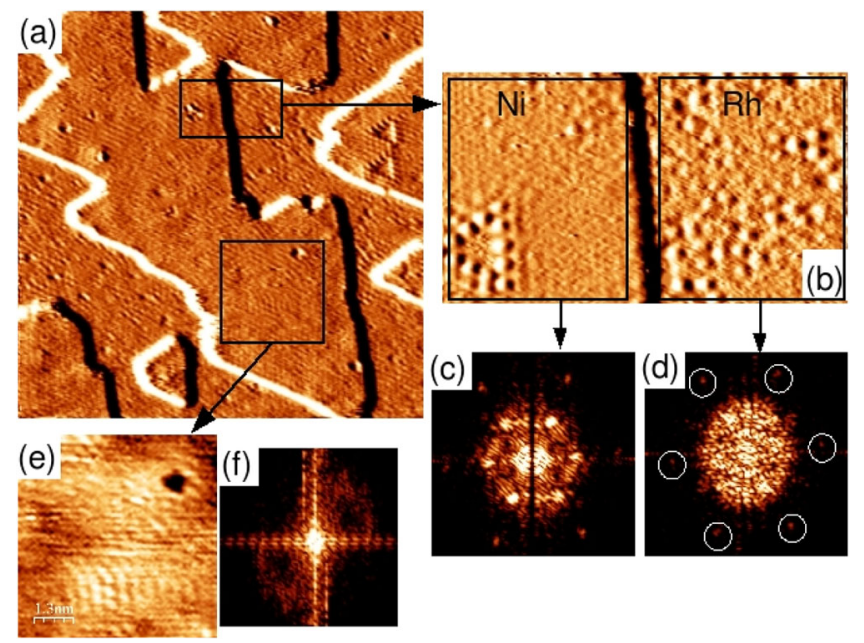

FIG. 1 (color online). (a) Derivative of a $30 \times 27 \mathrm{~nm}^{2}$ STM image $(30 \mathrm{mV} ; 14 \mathrm{pA})$, showing the $\mathrm{SW}$ on the Ni adislands and the $\mathrm{Rh}(111)$. (b) Zoom in of (a) showing atomic resolution. (c) FT of the Ni area of (b) and (d) FT of the Rh area of (b), both are calculated from the original STM image. (e) STM image of the SW on the Ni ad-island and (f) its FT showing 2 arcs. 
ruled out [16]. A full characterization of the growth of the Ni films is outside the purpose of this Letter; an appropriate analysis is given in a dedicated report [17].

Figure 1(a) presents the derivative of a STM image of Ni adislands on the $\mathrm{Rh}(111)$ substrate for the $0.8 \mathrm{ML}$ coverage, acquired at a voltage of $30 \mathrm{mV}$. Under these conditions, STM images reflect approximately the variation of the LDOS at $E_{F}$ [10].

The image shows the presence of a strong interference pattern on both the $\mathrm{Rh}(111)$ substrate and the $\mathrm{Ni}$ adislands. Similar patterns were observed at LT for Co and Ni deposited on $\mathrm{Cu}(111)[18,19]$ and at $\mathrm{RT}$ for $\mathrm{Au}(111)[13,20]$ and $\mathrm{Cu}(111)$ [21]. On the adislands, confinement enhances the interference and the SWs can be imaged with STM at RT; see Fig. 1(e). By taking the Fourier transform (FT) of the STM image [22] the wave vector $k$ of the SW is estimated.

A zoom in of the image is shown in Fig. 1(b), and Figs. 1(c) and 1(d) present the FT of the Ni and Rh areas, respectively. The FT spectrum of the Rh area, Fig. 1(d), shows a disc and 6 faint spots around the center, highlighted by the circles. The spots are given by the periodicity of the surface atoms and can be used to calibrate the image. The disc is given by the Friedel oscillations, resulting from interference of all the wave vectors between 0 and $k$, where $k$ is the disc's radius. An average measure of $k$ returns a value of $\sim 1.3 \AA^{-1}$. A value of $k_{F}$ for $\mathrm{Rh}(111)$ from early measurements of the de Haas and van Alphen effect [23] in Rh gave values of the radius vector of the Fermi surface along the $\Gamma-L$ line of 0.65 and $0.69 \AA^{-1}$ while angle-resolved photoemission spectroscopy on $\mathrm{Rh}$ (111) from Di et al. [24], reported a value for $k_{\|}=$ $0.70 \AA^{-1}$ along the $\Gamma-K$ line and $0.76 \AA^{-1}$ along the $\Gamma-M$ line. Using an average we estimate the Fermi vector to be $k_{F} \sim 0.7 \AA^{-1}$. A value of $k \sim 1.3 \AA^{-1}$ measured for the experimental SW is comparable with $2 k_{F} \sim 1.4 \AA^{-1}$ estimated from literature, suggesting that the SWs are Friedel oscillations of $\mathrm{Rh}$ electrons.

In the case of the $\mathrm{Ni}$ adislands one has to distinguish between two cases, namely, with or without the triangular clusters on them. Both cases are presented in Fig. 1, that shows the STM derivative in (b) and the FT in (c) with a cluster and the STM in (e) and the FT in (f) of the SW pattern without the clusters. In the latter case the SW pattern, see Fig. 1(e), only contains a wavevector component. This is confirmed in the FT of the image, see Fig. 1(f), that shows an incomplete circle positioned around the center of the spectrum, indicating that the SW pattern is given by energy-resolved Friedel oscillations [22]. From Fig. 1(e), it can be intuitively understood that the SW pattern on the $\mathrm{Ni}$ island is given by 2 waves scattered in 2 directions resulting in the FT spectrum showing 2 arcs of an incomplete circle. The radius of the arcs is, as in the case of the Rh disc, $\sim 1.3 \AA^{-1}$.

However, in the presence of the clusters, the FT image shows two sets of 6 spots [Fig. 1(c)]. The spots further away from the center are given by the periodicity of the surface atoms while the spots closer to it are given by the cluster. A superposition of the FT images of the Ni and the $\mathrm{Rh}$, presented in Figs. 1(c) and 1(d) respectively, shows a very good correspondence between the two sets of 6 spots given by the surface atoms and also between the disc of the $\mathrm{Rh}$ and the spots given by the triangular cluster of the $\mathrm{Ni}$. This confirms the LEED data, i.e., that the $\mathrm{Ni}$ is fully stretched by the Rh surface and also indicates that the SW patterns from the Ni islands and the Rh surface are related. Moreover, the spots produced by the cluster are located around the same $k$ of the arcs given by the SW, which indicates that the adatoms and the SW have similar periodicity.

Figure 1(b) shows one of the clusters on top of the $\mathrm{Ni}$ adisland. Remarkably, the cluster appears "hollow", meaning that it is composed entirely of adatoms positioned along its perimeter. Clusters of this type were consistently measured in the majority of $\mathrm{Ni}$ adislands and they showed a very regular size and shape; see Fig. 2. Statistically, see the histogram in Fig. 2(a), the largest number of clusters were composed by either 12,15 , or 18 adatoms, corresponding to triangles of sides equal, respectively, to 20,25 , or $30 \AA$. AES data showed the presence of only $\mathrm{Ni}$ and $\mathrm{Rh}$ species.

A zoom in on one of the clusters is presented in Fig. 2(c). The separation of the adatoms is $4.99 \pm 0.05 \AA$ and the corrugation is $0.42 \pm 0.05 \AA$.

Friedel oscillations modify the adsorption properties of the surface. The variation of the LDOS ( $\Delta$ LDOS) due to Friedel oscillations depends on $k_{F}$, the distance $r$ from the scattering center and the Fermi level phase shift $\delta$, which
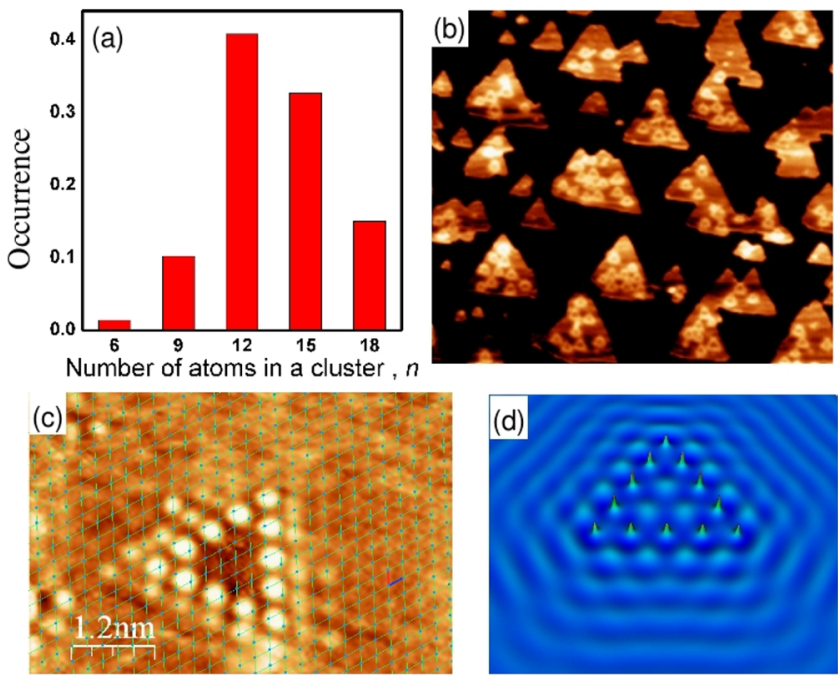

FIG. 2 (color online). (a) Distribution of the clusters size vs the number of atoms in it. (b) $90 \times 80 \mathrm{~nm}^{2}$ STM image $(30 \mathrm{mV}$; $15 \mathrm{pA}$ ), showing the $\mathrm{Ni}$ island with the clusters on top. (c) $5.5 \times 3.6 \mathrm{~nm}^{2} \mathrm{STM}$ image $(30 \mathrm{mV} ; 14 \mathrm{pA})$ that shows one cluster and an hexagonal lattice superimposed on the surface atoms. (d) $\triangle$ LDOS map obtained by linear combination of the single patterns produced by each interfering adatom. 
determines the position of the first LDOS maximum, and is described by the equation [10]:

$$
\Delta \mathrm{LDOS} \propto \frac{1}{k_{F} r}\left[\cos ^{2}\left(k_{F} r-\frac{\pi}{4}+\delta\right)-\cos ^{2}\left(k_{F} r-\frac{\pi}{4}\right)\right] .
$$

A maximum in $\triangle$ LDOS is a potential well and it is a suitable adsorption site. Equation (1) can be used to draw a map of the $\Delta$ LDOS around a single atom. In the case where one considers more than one atom, the resulting interference pattern is given by a linear combination of the single patterns produced by each interfering adatom.

Figure 2(d) shows the calculated map of the $\Delta$ LDOS for the case of a cluster of 12 atoms. This image can be directly compared with the STM in Fig. 2(c). Neglecting the atomic resolution, that was not included in the model, the images are very similar, in particular, in the immediate vicinity of the cluster, where maxima and minima in the $\triangle$ LDOS, due to quantum interference, alternate regularly. This simple model gives an indirect confirmation that the pattern we see is indeed due to Friedel oscillations and that most likely the oscillations are responsible for the formation of the clusters.

To understand the self-assembly of the clusters, we studied the adsorbate-adsorbate interaction energy $\Delta U_{\text {int }}$ for 2 adatoms separated by a distance $r$ and interacting via Friedel oscillations, which is given by $[9,25,26]$ :

$$
\Delta U_{\mathrm{int}} \simeq-\left(\frac{2 \sin \delta}{\pi}\right)^{2} \frac{\sin \left(2 k_{F} r+2 \delta\right)}{\left(k_{F} r\right)^{2}},
$$

where $\delta$ gives information on the scattering properties of the atoms and has a direct effect on the position of the first minimum in $\Delta U_{\text {int }}$, that corresponds to the equilibrium distance between the interacting adatoms [6,9]. If we put the $k_{F}$ of $\mathrm{Rh}(111)$ into our analysis, in order to match our experimental data a minimum at $r=4.95 \AA$ is obtained for a value of $\delta=0.1 \pi$. The value of the phase shift for a perfect scatterer is $\delta=0.5 \pi$. A value of $\delta=0.1 \pi$ means that the $\mathrm{Ni}$ atoms in the adislands are quite ineffective scatterers. This may be a consequence of the strain present in the adislands that places the $\mathrm{Ni}$ atoms further apart, altering their scattering properties. However, it has to be noted that this value of $\delta$ carries a large error and that an adequate evaluation requires accurate fitting of LT experimental data, in order to assume a quantitative significance.

The interaction energy $\Delta U_{\text {int }}$ described in the Eq. (2) can be used, not only to calculate the preferred adsorption site, but once two atoms join to form a dimer, the $\Delta U_{\text {int }}$ around them can be evaluated by a linear combination of the contributions of each atom. The result is presented in the inset of Fig. 3(a), that shows a 3D map of the $\Delta U_{\text {int }}$ around a dimer, shown in blue. The positions of the lowest minima are shown in white. Thus, if the third atom is placed at the site marked in white, this should form a cluster of atoms situated at the minimum energy sites formed for each of

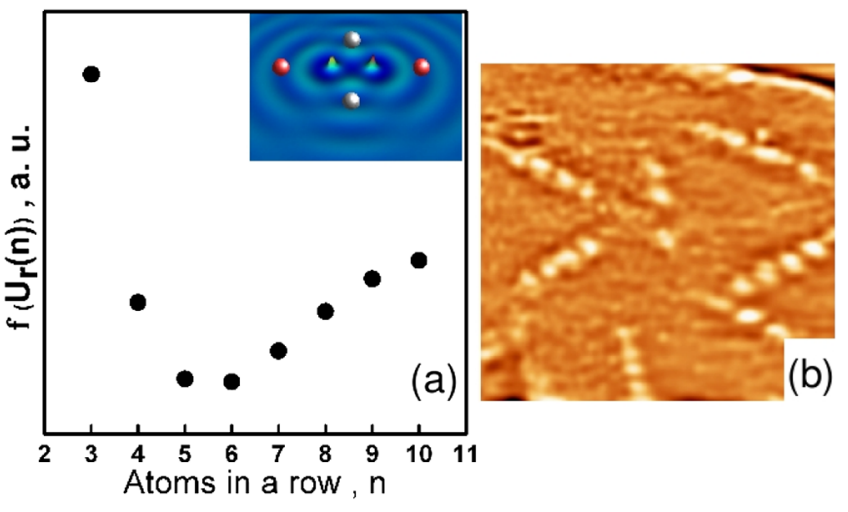

FIG. 3 (color online). (a) Plot of $f\left(U_{r}(n)\right)$ showing a minimum for rows of 5 and 6 adatoms. (inset) 3D map of the $\Delta U_{\text {int }}$ around a dimer. (b) $5.8 \times 5.5 \mathrm{~nm}^{2} \mathrm{STM}$ image $(38 \mathrm{mV} ; 5 \mathrm{pA})$ of the atomic rows on top of a $\mathrm{Ni}$ ad-island.

them by the other two. Indeed, clusters of 3 adatoms were observed by us. Other areas of minimum energy are found along the axis that joins the two atoms, on both sides, that correspond to suitable adsorption sites, marked red in the figure. These minima are given by the combination of the first minimum in $\Delta U_{\text {int }}$ of one atom with the second minimum of the other atom.

A similar calculation can be performed to obtain the $\Delta U_{\text {int }}$ by means of Friedel oscillations for rows of any length. To estimate the length of the most stable row we calculate the function $f\left(U_{r}(m)\right)=2 U_{r}(m)-U_{r}(m-$ $1)-U_{r}(m+1)$, where $m$ is the number of atoms in a row. The function $f\left(U_{r}(m)\right)$ measures the relative energy gain or loss when two rows exchange individual atoms. The resulting graph is presented in Fig. 3(a) and shows that the most stable rows have a length of 5 or 6 adatoms. This is in good agreement with the STM data, as rows of either 4,5 , or 6 adatoms were imaged on several occasions; see Fig. 3(b).

Based on this evidence, it is possible to speculate that the relatively stable rows migrate on the surface interacting with each other to form the triangular clusters. Once again, with a linear combination of the potential energies of each atom, we can calculate the interaction energy in the case of a cluster, $U_{t}(n)$ with $n$ being the number of atoms in the cluster and compare it with the cumulative interaction energy of three separate rows with the same number of atoms in total, calculating the function $l\left(U_{r}(m), U_{t}(n),\right)=$ $l_{m, n}=U_{t}(n)-3 U_{r}(m)$ for $n=3 m$. The plot of $l_{m, n}$ versus the total number of adatoms $n$ in a cluster, is presented in Fig. 4(a). This shows that clusters of 12,15 , or 18 atoms are more stable with respect to 3 separate rows having an equivalent number of atoms as the absolute value of $l_{m, n}$ is negative, in excellent agreement with our experimental data. In Fig. 4(b) we give a model of the possible sites of adsorption of the adatoms in a cluster of 12 adatoms, according to the STM image presented in Fig. 2(c). In 


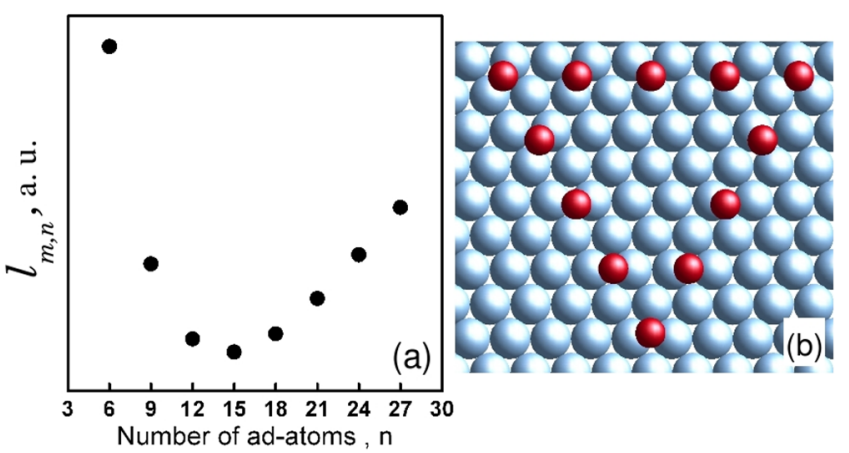

FIG. 4 (color online). (a) Plot of $l_{m, n}$ showing a minimum for clusters of 12, 15, and 18 atoms. (b) Model of the likely sites of adsorption for a cluster of 12 atoms. Light blue (light gray) corresponds to the Ni layer while red (dark gray) represents the adatoms of the cluster.

the figure, light blue corresponds to the Ni layer while red is used for the $\mathrm{Ni}$ atoms of the cluster.

From the STM image in Fig. 2(b) one can make another interesting observation. The clusters on the $\mathrm{Ni}$ adislands tend to stay close together. Thus, it seems that the clusters interact with each other similarly to the way the adatoms interact with each other. The map presented in Fig. 2(d), shows the alternation of maxima and minima of the $\Delta$ LDOS around the cluster, which are associated with the regions of low and high potential energy, respectively. The presence of potential wells can either induce the formation of new clusters or the diffusion of whole clusters on the surface in order to maximize their density.

In conclusion, we have presented a study on the selfassembly of hollow equilateral triangular nanoclusters of narrow range of sizes at RT. We put forward a model that explains the formation of the clusters on the basis of interaction of the adatoms via Friedel oscillations. We stress the importance of the enhancement of the interference patterns by confinement of the SW to adislands leading to the RT assembly. The modeled pattern of the $\Delta$ LDOS in the vicinity of the cluster showed an excellent correlation with the experimental data. We demonstrated that, according to our model, the clusters consisting of 12 , 15 or 18 atoms do correspond to structures of minimum energy. We further demonstrate that a separate row can be more stable if it consists of a particular number of adatoms, namely, 5 or 6 , as observed experimentally. The attractive interaction between the clusters, that tend to form in the vicinity of each other, can also be explained on the basis of $\Delta$ LDOS maps formed by Friedel interference patterns from the individual adatoms. Using a comparison of our model with experimental results we have calculated the phase shift of the surface Friedel oscillations, $\delta \sim 0.1 \pi$, indicating that the $\mathrm{Ni} / \mathrm{Rh}(111)$ system has weak scattering properties.

The authors acknowledge Science Foundation Ireland (SFI) for funding provided under the contract MASF185.

*gmanai@tcd.ie

[1] D. Eigler and E. Schweizer, Nature (London) 344, 524 (1990).

[2] V.S. Stepanyuk et al., Phys. Rev. B 68, 205410 (2003).

[3] H. F. Ding et al., Phys. Rev. B 76, 033409 (2007).

[4] M. F. Crommie et al., Science 262, 218 (1993).

[5] V. S. Stepanyuk et al., New J. Phys. 9, 388 (2007).

[6] F. Silly et al., Phys. Rev. Lett. 92, 016101 (2004).

[7] N. Memmel and E. Bertel, Phys. Rev. Lett. 75, 485 (1995).

[8] W. Shockley, Phys. Rev. 56, 317 (1939).

[9] P. Hyldgaard and M. Persson, J. Phys. Condens. Matter 12, L13 (2000).

[10] M.F. Crommie et al., Nature (London) 363, 524 (1993).

[11] A. Zangwill, Physics at Surfaces (Cambridge University Press, Cambridge, England, 1988).

[12] P. T. Sprunger et al., Science 275, 1764 (1997).

[13] Y. Hasegawa and P. Avouris, Phys. Rev. Lett. 71, 1071 (1993).

[14] A. Wander et al., Surf. Sci. 281, 42 (1993).

[15] S. Ovesson et al., Phys. Rev. Lett. 83, 2608 (1999).

[16] J. Wu et al., Phys. Rev. Lett. 89, 146103 (2002).

[17] G. Manai et al. (unpublished).

[18] S. Pons, Phys. Rev. B 64, 193408 (2001).

[19] L. Diekhöner et al., Phys. Rev. Lett. 90, 236801 (2003).

[20] P. Avouris and I. W. Lyo, Science 264, 942 (1994).

[21] S. L. Silva and F. M. Leibsle, Surf. Sci. 441, L904 (1999).

[22] L. Petersen et al., Phys. Rev. B 57, R6858 (1998).

[23] P. T. Coleridge, Proc. R. Soc. A 295, 458 (1966).

[24] W. Di, Phys. Rev. B 49, 4821 (1994).

[25] The evaluation of a 3-body interaction energy only increases the $\Delta U_{\text {(int) }}$ by a quarter [26], leaving its treatment qualitatively the same.

[26] P. Hyldgaard and T. Einstein, Europhys. Lett. 59, 265 (2002). 